\title{
SHORT-PERIOD $g$-MODE PULSATIONS IN LOW-MASS WHITE DWARFS TRIGGERED BY H-SHELL BURNING
}

\author{
A. H. Córsico ${ }^{1,2}$ AND L. G. Althaus ${ }^{1,2}$ \\ ${ }^{1}$ Grupo de Evolución Estelar y Pulsaciones, Facultad de Ciencias Astronómicas y Geofísicas, Universidad Nacional de La Plata, \\ Paseo del Bosque s/n, 1900 La Plata, Argentina; acorsico@ fcaglp.unlp.edu.ar \\ ${ }^{2}$ Instituto de Astrofísica La Plata, IALP (CCT La Plata), CONICET-UNLP, Paseo del Bosque s/n, 1900 La Plata, Argentina \\ Received 2014 July 17; accepted 2014 August 28; published 2014 September 8
}

\begin{abstract}
The detection of pulsations in white dwarfs with low mass offers the possibility of probing their internal structures through asteroseismology and placing constraints on the binary evolutionary processes involved in their formation. In this Letter, we assess the impact of stable $\mathrm{H}$ burning on the pulsational stability properties of low-mass He-core white dwarf models resulting from binary star evolutionary calculations. We found that besides a dense spectrum of unstable radial modes and nonradial $g$ and $p$ modes driven by the $\kappa$ mechanism due to the partial ionization of $\mathrm{H}$ in the stellar envelope, some unstable $g$ modes with short pulsation periods are also powered by $\mathrm{H}$ burning via the $\varepsilon$ mechanism of mode driving. This is the first time that $\varepsilon$ destabilized modes are found in models representative of cool white dwarf stars. The short periods recently detected in the pulsating low-mass white dwarf SDSS J111215.82+111745.0 could constitute the first evidence of the existence of stable $\mathrm{H}$ burning in these stars, in particular in the so-called extremely low-mass white dwarfs.
\end{abstract}

Key words: stars: evolution - stars: interiors - stars: oscillations - white dwarfs

Online-only material: color figures

\section{INTRODUCTION}

Low-mass $\left(M_{\star} / M_{\odot} \lesssim 0.45\right)$ white dwarfs (WDs) are likely the result of intense mass-loss events at the red giant branch stage of low-mass stars in binary systems before the He flash onset (Althaus et al. 2010). Since the He flash does not occur, their cores must be made of $\mathrm{He}$, at variance with average mass $\left(M_{\star} \sim 0.6 M_{\odot}\right)$ WDs, which are thought to have $\mathrm{C} / \mathrm{O}$ cores. In particular, binary evolution is the most likely origin for the so-called extremely low-mass (ELM) WDs, which have masses below $\sim 0.18-0.20 M_{\odot}$. According to detailed evolutionary computations (Althaus et al. 2001, 2013), ELM WDs must harbor very thick $\mathrm{H}$ envelopes that are able to sustain residual $\mathrm{H}$ nuclear burning via the $p p$-chain, leading to markedly long evolutionary timescales.

Recently, numerous low-mass WDs, including ELM WDs, have been detected through the ELM survey and the SPY (ESO SN Ia Progenitor surveY) and WASP surveys (see Koester et al. 2009; Brown et al. 2010, 2012; Maxted et al. 2011; Kilic et al. 2011, 2012). The interest in low-mass WDs has been greatly boosted by the recent discovery that some of them pulsate (Hermes et al. 2012, 2013a, 2013b). The discovery of pulsating low-mass WDs constitutes an unprecedented opportunity for probing their interiors and eventually testing their formation channels by employing the tools of asteroseismology. A few theoretical pulsational analysis of these stars have been performed previously, which have yielded very interesting results. In particular, it has been shown that $g$ modes in ELM WDs are restricted mainly to the core regions and $p$ modes to the envelope, providing the opportunity to constrain both the core's and the envelope's chemical structure of these stars via asteroseismology (Steinfadt et al. 2010; Córsico et al. 2012). Also, many unstable $g$ and $p$ modes excited by the $\kappa$ mechanism at roughly the right effective temperatures and the correct range of the periods observed in pulsating low-mass WDs have been found by Córsico et al. (2012), and later confirmed by Van Grootel et al. (2013).
In this Letter, we perform a new pulsation stability analysis on the recently published set of state-of-the-art evolutionary models of low-mass He-core WDs of Althaus et al. (2013). Here we focus on the role of stable $\mathrm{H}$ burning on the driving of pulsations through the $\varepsilon$ mechanism. In this excitation mechanism, the driving is due to the strong sensitivity of nuclear burning on temperature (Unno et al. 1989; Gautschy \& Saio 1995). Our computations show that in addition to the existence of a dense spectrum of unstable radial, $g$, and $p$ modes driven by the $\kappa$ mechanism due to the partial ionization of $\mathrm{H}$, some unstable short-period $g$ modes of low radial order exist that are mainly destabilized by H burning via the $\varepsilon$ mechanism. Recently, Maeda \& Shibahashi (2014) reported the existence of low-order $g$ modes destabilized by the $\varepsilon$ mechanism in hot H-rich preWD models. The results of the present Letter constitute the first theoretical evidence of pulsation modes excited by the $\varepsilon$ mechanism in cool WD stars.

\section{EVOLUTIONARY MODELS AND NUMERICAL TOOLS}

Realistic configurations for the low-mass He-core WD models employed in this work were obtained by Althaus et al. (2013) with the LPCODE evolutionary code by mimicking the binary evolution of progenitor stars. Specifically, the models were derived by computing the non-conservative evolution of a binary system consisting of an initially $1 M_{\odot}$ zero-age main sequence star and a $1.4 M_{\odot}$ neutron star for various initial orbital periods. Details about this procedure can be found in that paper. Since $\mathrm{H}$-shell burning is the main source of star luminosity during most of the evolution of ELM WDs, the computation of realistic initial WD structures is a fundamental issue, in particular concerning the correct assessment of the $\mathrm{H}$ envelope mass left by progenitor evolution. We analyzed six sequences with stellar masses of $M_{\star} / M_{\odot}=0.1554,0.1650,0.1762,0.1806,0.2707$, and 0.4352 . The pulsation computations were performed with the help of the linear, radial, and nonradial, nonadiabatic versions of the LP-PUL pulsation code described in Córsico 


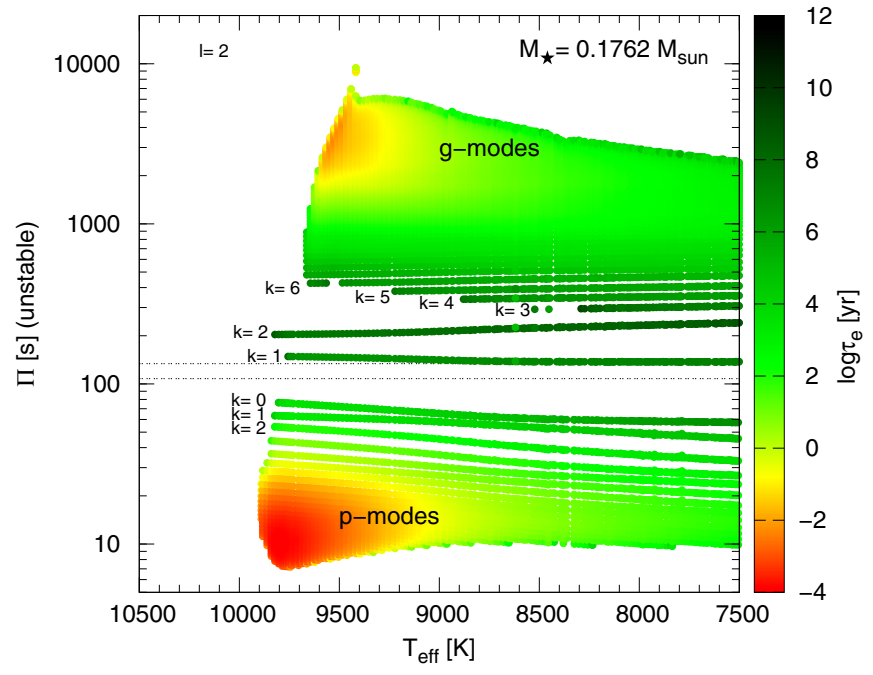

Figure 1. Unstable $\ell=2$ mode periods $(\Pi)$ in terms of the effective temperature corresponding to an ELM WD model sequence with $M_{\star}=0.1762 M_{\odot}$. Color coding indicates the value of the logarithm of the $e$-folding time $\left(\tau_{e}\right)$ of each unstable mode (right scale). Horizontal dashed lines correspond to the short periods observed in the pulsating ELM WD star SDSS J111215.82+111745.0, at $\Pi \sim 108 \mathrm{~s}$ and $\Pi \sim 134 \mathrm{~s}$.

(A color version of this figure is available in the online journal.)

et al. (2006; see also Córsico et al. 2009). We have considered $\ell=1$ and $\ell=2$ modes. Our computations ignore the perturbation of the convective flux; that is, we assume the "frozen-in convection" approximation.

\section{NONADIABATIC RESULTS}

In Figure 1, we depict the instability domain of $\ell=2$ periods in terms of the effective temperature for the ELM WD model sequence with $M_{\star}=0.1762 M_{\odot}$. The palette of colors (right scale) indicates the value of the logarithm of the $e$-folding time (in years) of each unstable mode, defined as $\tau_{\mathrm{e}}=1 /|\Im(\sigma)|$, where $\Im(\sigma)$ is the imaginary part of the complex eigenfrequency $\sigma$. Many unstable high-order pulsation modes, which are clearly grouped in two separated regions, exist, one of them characterized by long periods and associated with $g$ modes, and the other one characterized by short periods and corresponding to $p$ modes. Unstable radial modes (not shown in the figure) are also found. Most of these modes are destabilized by the $\kappa$ mechanism acting at the surface $\mathrm{H}$ partial ionization zone. The strongest excitation (that is, the smallest $e$-folding times, red zones) is found for high-order $g$ and $p$ modes, with periods in the ranges (2000-6000) s and (10-30) s, respectively, and effective temperatures near the hot boundary of the instability islands $\left(T_{\mathrm{eff}} \sim 9700 \mathrm{~K}\right)$. Similar results, although with longer unstable $g$-mode periods (3000-10,000) $\mathrm{s}$, are obtained for $\ell=1$ (not shown). At lower effective temperatures, these unstable modes become less excited, as reflected by the higher values of the $e$-folding times (100-10,000 $\mathrm{yr})$. On the other hand, low-order $g$ and $p$ modes and even the $f$ mode are also driven, although they take much longer to become unstable, as it is reflected by the dark green tone in the figure $\left(\tau_{e} \sim 10^{6}-10^{9} \mathrm{yr}\right)$. However, since the evolution of the ELM WDs is so slow, these modes would still have enough time to get excited and reach observable amplitudes. This is confirmed by examining Table 1 , in which we show the time $\Delta t$ that the models take to cool from $T_{\text {eff }} \sim 10,000 \mathrm{~K}$ to $\sim 8000 \mathrm{~K}$, and the maximum $e$-folding times of the unstable short-period $g$
Table 1

The Stellar Mass, the Mass of $\mathrm{H}$, the Evolutionary Timescale, the Radial Order and Harmonic Degree, the $T_{\text {eff }}$ Range of Instability, the Average Period, and the Maximum $e$-folding Time of Unstable Short-period $\ell=1,2 \mathrm{~g}$ Modes Destabilized Through the $\epsilon$ Mechanism

\begin{tabular}{|c|c|c|c|c|c|c|}
\hline $\begin{array}{l}M_{\star} \\
\left(M_{\odot}\right)\end{array}$ & $\begin{array}{c}M_{\mathrm{H}} / M_{\star} \\
\left(10^{-3}\right)\end{array}$ & $\begin{array}{c}\Delta t \\
\left(10^{9} \mathrm{yr}\right)\end{array}$ & $k(\ell)$ & $\begin{array}{l}T_{\text {eff }} \\
(\mathrm{K})\end{array}$ & $\begin{array}{c}\langle\Pi\rangle \\
(\mathrm{s})\end{array}$ & $\begin{array}{c}\tau_{e}^{\max } \\
\left(10^{9} \mathrm{yr}\right) \\
\end{array}$ \\
\hline \multirow[t]{5}{*}{0.1554} & 25.4 & 3.13 & $2(1)$ & $\lesssim 8500$ & 350 & 0.07 \\
\hline & & & $3(1)$ & $9000-8300$ & 470 & 0.97 \\
\hline & & & $2(2)$ & $\lesssim 8100$ & 227 & 0.12 \\
\hline & & & $3(2)$ & $8600-8360$ & 291 & 0.2 \\
\hline & & & $4(2)$ & $9000-8800$ & 355 & 0.33 \\
\hline \multirow[t]{6}{*}{0.1650} & 18.7 & 5.53 & $1(1)$ & $\lesssim 8200$ & 250 & 0.07 \\
\hline & & & $2(1)$ & $\lesssim 9500$ & 340 & 0.17 \\
\hline & & & $3(1)$ & $950-09020$ & 450 & 1.3 \\
\hline & & & $4(1)$ & $\lesssim 7800$ & 580 & 0.8 \\
\hline & & & $2(2)$ & $\lesssim 8950$ & 214 & 0.5 \\
\hline & & & $3(2)$ & 9400-9300 & 277 & 0.5 \\
\hline \multirow[t]{8}{*}{0.1762} & 14.5 & 7.56 & $1(1)$ & $\lesssim 9100$ & 247 & 1.4 \\
\hline & & & $2(1)$ & $\lesssim 10000$ & 320 & 0.2 \\
\hline & & & $3(1)$ & $\lesssim 8700$ & 470 & 0.7 \\
\hline & & & $4(1)$ & $8900-8700$ & 550 & 0.09 \\
\hline & & & $5(1)$ & 9200-9150 & 620 & 0.06 \\
\hline & & & $1(2)$ & $\lesssim 8300$ & 140 & 0.02 \\
\hline & & & $2(2)$ & $\lesssim 9900$ & 220 & 0.4 \\
\hline & & & $3(2)$ & $8300-7700$ & 297 & 0.25 \\
\hline \multirow[t]{3}{*}{0.1806} & 3.68 & 0.34 & $1(1)$ & $\lesssim 10500$ & 270 & 0.05 \\
\hline & & & $2(1)$ & $10200-9700$ & 355 & 0.02 \\
\hline & & & $1(2)$ & $\lesssim 10500$ & 178 & 0.4 \\
\hline 0.2707 & 1.09 & 0.33 & $\cdots$ & $\cdots$ & $\cdots$ & $\cdots$ \\
\hline \multirow[t]{2}{*}{0.4352} & 0.63 & 0.91 & $1(1)$ & $\lesssim 9950$ & 137 & 0.12 \\
\hline & & & $1(2)$ & $\lesssim 10000$ & 80 & 0.15 \\
\hline
\end{tabular}

modes for each stellar mass considered in this work. Note that in all cases, the $e$-folding times are substantially shorter than the time that models spent evolving in the regimen of interest. In particular, for $M_{\star}=0.1762 M_{\odot}$, the maximum $e$-folding times are a factor 5-100 shorter than the evolutionary timescale for the $g$ modes with $k=1, \ldots, 5$.

At this stage, we can wonder what is the role (if any) of stable $\mathrm{H}$ burning in the destabilization of the modes shown in Figure 1. To answer this query, we have repeated the stability computations, but this time by consistently suppressing the action of this destabilizing agent, that is, by forcing the nuclear energy production rate, $\varepsilon$, and their logarithmic derivatives $\varepsilon_{\mathrm{T}}=(\partial \ln \varepsilon / \partial \ln T)_{\rho}$ and $\varepsilon_{\mathrm{T}}=(\partial \ln \varepsilon / \partial \ln \rho)_{\mathrm{T}}$ to be zero in the pulsation equations. The results are shown in Figure 2. Interestingly enough, the $k=2 g$ mode becomes stable and do not appear in this plot. Something similar happens with the modes $k=1$ and $k=3$ in certain ranges of $T_{\text {eff }}$. We can conclude that these modes are excited (at least in part) by the $\varepsilon$ mechanism through the H-burning shell.

In what follows, we focus our discussion on a template model at $T_{\text {eff }}=8250 \mathrm{~K}$. Figure 3 displays the normalized $\ell=1$ and $\ell=2$ growth rates $\eta=-\Re(\sigma) / \Im(\sigma)(\Re(\sigma)$ being the real part of the complex eigenfrequency $\sigma$ ) in terms of the pulsation periods for this model. $\eta>0(\eta<0)$ implies unstable (stable) modes. The large black (small red) dots connected with continuous (dashed) lines correspond to the case where the $\varepsilon$ mechanism is explicitly considered (suppressed) in the nonadiabatic calculations. For $\ell=1$, the range of periods of unstable $g$ modes is $234-5628 \mathrm{~s}(1 \leqslant k \leqslant 57)$, while for $\ell=2$ 


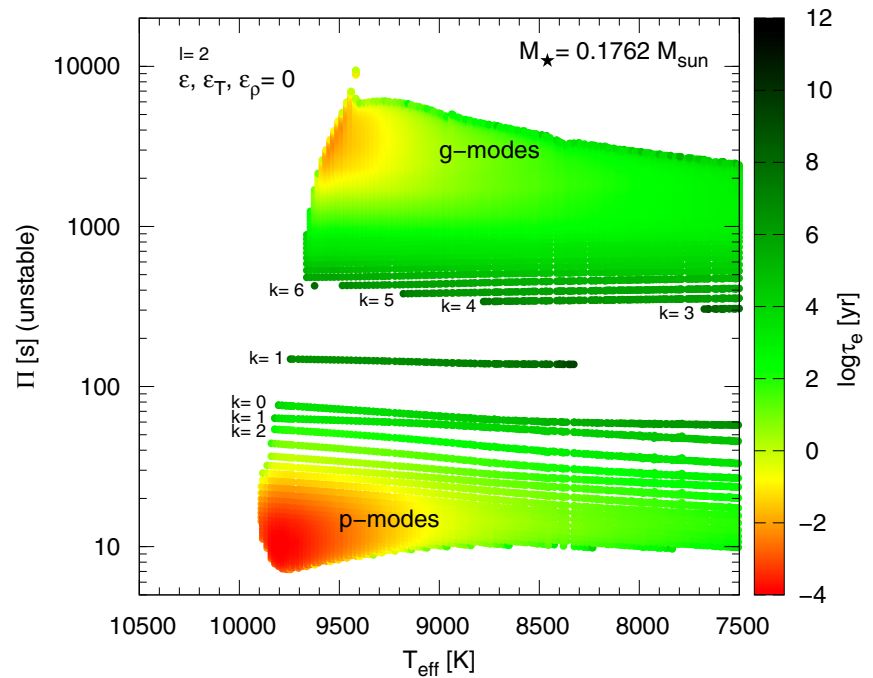

Figure 2. Same as Figure 1, but for the case in which the effects of the $\varepsilon$ mechanism are suppressed in the stability calculations.

(A color version of this figure is available in the online journal.)

the periods of unstable modes are in the range 137-3217 s $(1 \leqslant k \leqslant 56)$. In the absence of the destabilizing effect of the $\varepsilon$ mechanism, the unstable modes with $k=1,2$, and 3 and for both values of the harmonic degree $(\ell=1$ and 2$)$ turn out to be stable, while the remainder modes of the pulsation spectrum $(k \geqslant 4)$ remain unchanged. Clearly, the destabilizing effect of $\mathrm{H}$ burning is crucial for the modes with $k=1,2$, and 3 to be unstable. Interestingly enough, the period of the $\ell=2, k=1$ mode $(\sim 137 \mathrm{~s})$ is very close to one of the short periods observed in the pulsating ELM WD SDSS J111215.82+111745.0 (Hermes et al. 2013b), $\Pi \sim 134 \mathrm{~s}$ (see the rightmost vertical gray line in Figure 3). While the star is hotter than our models with the $\ell=2, k=1 \varepsilon$ destabilized mode $(\sim 9590 \mathrm{~K}$ versus $\sim 8300 \mathrm{~K})$, it should be kept in mind that the spectroscopic estimate of the effective temperature for this star (like for other ELM WDs) can suffer from rather large uncertainties.
In the left panels of Figure 4, we show the Lagrangian perturbation of temperature, $\delta T / T$, for the $\ell=2$ modes with $k=1$ and $k=4$ of the template model. The peak of the (scaled) nuclear generation rate $\varepsilon$ at $r / R \sim 0.57$ marks the location of the $\mathrm{H}$-burning shell at the $\mathrm{He} / \mathrm{H}$ chemical interface. We emphasize the position of the outermost maximum of $\delta T / T$ with a black dot. The $\varepsilon$ mechanism behaves as an efficient filter of modes that provides substantial driving only to those $g$ modes that have their largest maximum of $\delta T / T$ in the narrow region of the burning shell (Kawaler et al. 1986). For our template model, this condition is met by the modes with $k=1,2$, and 3 . As $k$ increases, the final extremum in $\delta T / T$ moves out of the burning shell. This is the case of the mode with $k=4$, which is not destabilized by the H-burning shell at all. The regions of the model that contribute to driving and damping for each of the selected modes can be drawn with the help of the differential work functions, $d W(r) / d r$, which are depicted in the middle panels of Figure 4. $d W(r) / d r>0$ implies driving, and $d W(r) / d r<0$ correspond to damping. If the $\varepsilon$ mechanism is allowed to operate, there is substantial driving for the $k=1$ mode at the location of the H-burning shell, and the same occurs for the $k=2$ and 3 modes (not shown). At variance with this, the mode with $k=4$ experiences some damping at that regions. When we set $\varepsilon$ and its derivatives to zero (red curves), strong damping takes place at those regions for modes with $k=1,2$, and 3, although the situation for the mode with $k=4$ does not change. In the right panels of Figure 4, we show $d W(r) / d r$ and the running work integral, $W(r)$, in terms of the coordinate $-\log \left(1-M_{r} / M_{\star}\right)$ which strongly amplifies the outer regions of the model. This allows us to investigate what happens in the outer part of the star, where the $\kappa$ mechanism operates due to the partial ionization of H. Notably, there exists strong driving for the two depicted modes due to the $\kappa$ mechanism at those regions (something that is barely distinguishable in the middle panels because this driving takes place at $r / R \sim 1$ ). Regarding the running work integral, we note that for the mode with $k=1, W(r)$ suddenly increases at the driving region where the H-burning shell is located, and also at the outer driving zone associated with the $\kappa$ mechanism. The combined effect of both driving regions overcomes the radiative damping from other
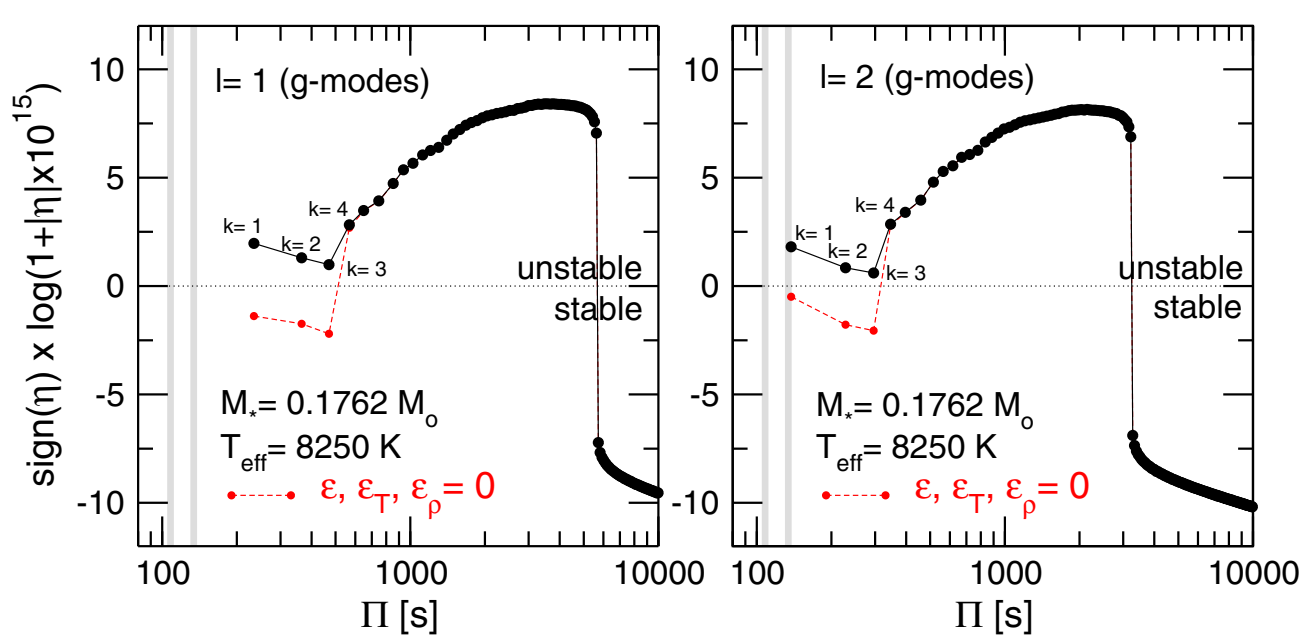

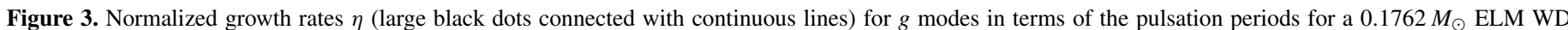

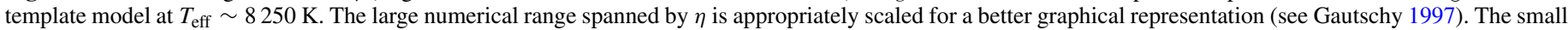

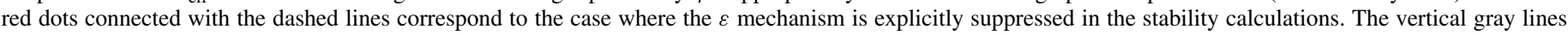
correspond to the short periods observed in the pulsating ELM WD star SDSS J111215.82+111745.0, at $\Pi \sim 108 \mathrm{~s}$ and $\Pi \sim 134 \mathrm{~s}$.

(A color version of this figure is available in the online journal.) 

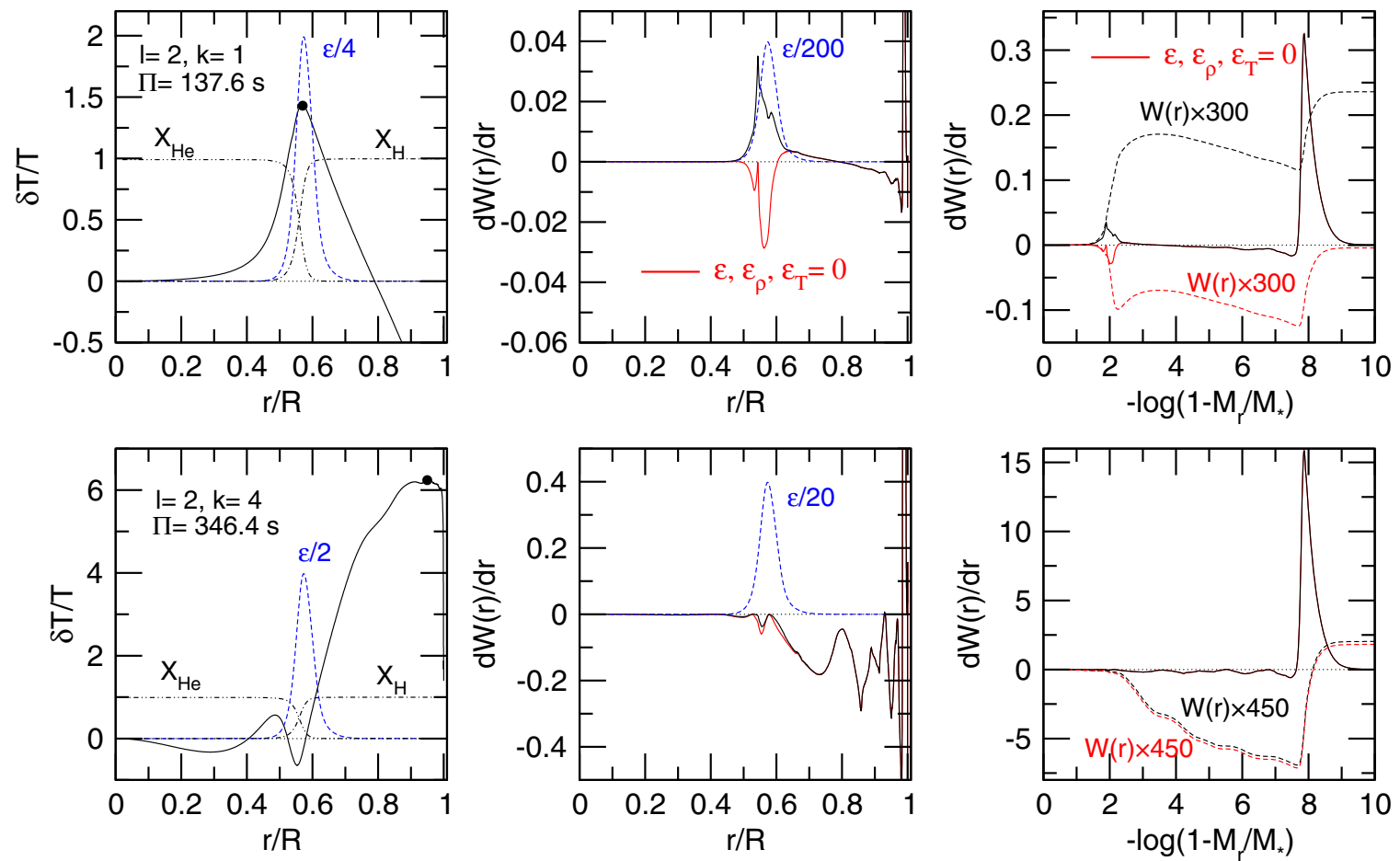

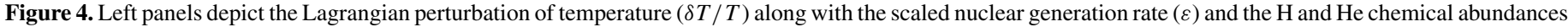

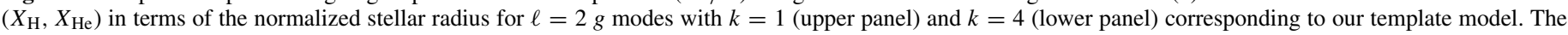

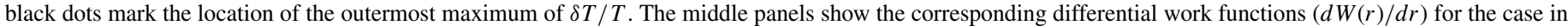

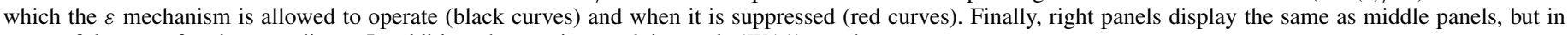
terms of the mass fraction coordinate. In addition, the running work integrals $(W(r))$ are shown.

(A color version of this figure is available in the online journal.)

parts of the star, and, as a result, this mode is globally unstable, as proven by the fact that $W>0$ at the stellar surface. The same holds for modes with $k=2$ and 3 . In the case of the mode with $k=4$, the damping effects at the H-burning shell and regions up to $-\log \left(1-M_{r} / M_{\star}\right) \sim 7$ are important, but not enough to overcome the strong driving at the outer regions, and so it is a unstable mode $(W(1)>0)$. If we inhibit the effects of the $\mathrm{H}$ burning (red dashed lines), the mode with $k=1$ becomes stable and the same occurs for the modes with $k=2$ and 3 . We can conclude that for this specific model, the modes with $k=1,2$, and 3 are globally unstable thanks to the destabilizing effect of the H-burning shell through the $\varepsilon$ mechanism.

\section{SUMMARY AND CONCLUSIONS}

In this work, we have shown for the first time that low-order short-period $g$ modes are destabilized through the $\varepsilon$ mechanism operating at the H-burning shell of cool low-mass He-core WD models. Note that the $\varepsilon$ mechanism is responsible for the fact that these modes become pulsationally unstable at early stages in which the $\kappa$ mechanism is still not efficient to drive pulsations (see Figure 1 in the particular case of the sequence with $M_{\star}=0.1762 M_{\odot}$ ). The $e$-folding times of the $\varepsilon$ destabilized modes are much shorter than the evolutionary timescale (Table 1), which means that they would have enough time to get excited and reach observable amplitudes. We find that in low-mass WDs, the range of periods destabilized by the $\varepsilon$ mechanism is $80 \lesssim \Pi \lesssim 600 \mathrm{~s}$; see Table 1 . In this connection, the pulsating ELM WD, SDSS J111215.82+111745.0 (Hermes et al. 2013b), that exhibits two short periods, at $\Pi \sim 108 \mathrm{~s}$ and $\Pi \sim 134 \mathrm{~s}$, constitutes an observational counterpart of these theoretically predicted unstable modes. Specifically, at the spectroscopic mass inferred for SDSS J111215.82+111745.0 (about $0.17 M_{\odot}$ ), our study predicts that the observed short periods, in particular that of $134 \mathrm{~s}$, is reproduced by the period of the excited mode with $\ell=2, k=1$ (see Table 1 and Figure 3 ), though our models with this mode destabilized by the $\varepsilon$ mechanism are admittedly cooler than the star. Although further exploration by considering stellar models with a range of $\mathrm{H}$ envelope masses, stellar masses, and effective temperatures, as well as higher degree $(\ell=3,4)$ modes are needed to be more conclusive, our results constitute the first evidence of the presence of low-order $g$ modes powered by the $\varepsilon$ mechanism in low-mass WDs, thus giving strong observational support to the existence of stable $\mathrm{H}$ burning in low-mass WDs.

Part of this work was supported by AGENCIA through the Programa de Modernización Tecnológica BID 1728/OC-AR, and by the PIP 112-200801-00940 grant from CONICET. This research has made use of NASA's Astrophysics Data System.

\section{REFERENCES}

Althaus, L. G., Córsico, A. H., Isern, J., \& García-Berro, E. 2010, A\&ARv, 18,471

Althaus, L. G., Miller Bertolami, M. M., \& Córsico, A. H. 2013, A\&A, 557, A19

Althaus, L. G., Serenelli, A. M., \& Benvenuto, O. G. 2001, MNRAS, 323, 471 Brown, W. R., Kilic, M., Allende Prieto, C., \& Kenyon, S. J. 2010, ApJ, 723, 1072

Brown, W. R., Kilic, M., Allende Prieto, C., \& Kenyon, S. J. 2012, ApJ, 744,142

Córsico, A. H., Althaus, L. G., \& Miller Bertolami, M. M. 2006, A\&A, 458, 259

Córsico, A. H., Althaus, L. G., Miller Bertolami, M. M., González Pérez, J. M., \& Kepler, S. O. 2009, ApJ, 701, 1008 
Córsico, A. H., Romero, A. D., Althaus, L. G., \& Hermes, J. J. 2012, A\&A, 547, A96

Gautschy, A. 1997, A\&A, 320, 811

Gautschy, A., \& Saio, H. 1995, ARA\&A, 33, 75

Hermes, J. J., Montgomery, M. H., Gianninas, A., et al. 2013a, MNRAS, 436, 3573

Hermes, J. J., Montgomery, M. H., Winget, D. E., et al. 2012, ApJL, 750, L28

Hermes, J. J., Montgomery, M. H., Winget, D. E., et al. 2013b, ApJ, 765, 102

Kawaler, S. D., Winget, D. E., Hansen, C. J., \& Iben, I., Jr. 1986, ApJL, 306, L41
Kilic, M., Brown, W. R., Allende Prieto, C., et al. 2011, ApJ, 727, 3

Kilic, M., Brown, W. R., Allende Prieto, C., et al. 2012, ApJ, 751, 141

Koester, D., Voss, B., Napiwotzki, R., et al. 2009, A\&A, 505, 441

Maeda, K., \& Shibahashi, H. 2014, arXiv:1405.4568

Maxted, P. F. L., Anderson, D. R., Burleigh, M. R., et al. 2011, MNRAS, 418, 1156

Steinfadt, J. D. R., Bildsten, L., \& Arras, P. 2010, ApJ, 718, 441

Unno, W., Osaki, Y., Ando, H., Saio, H., \& Shibahashi, H. 1989, Nonradial Oscillations of Stars (2nd ed.; Tokyo: Univ. Tokyo Press)

Van Grootel, V., Fontaine, G., Brassard, P., \& Dupret, M.-A. 2013, ApJ, 762, 57 